\title{
Communication
}

\section{Ionic Liquid Electrolytes for Safer and More Reliable Sodium Battery Systems}

\author{
Mariangela Bellusci, Elisabetta Simonetti ${ }^{D}$, Massimo De Francesco and \\ Giovanni Battista Appetecchi *
}

ENEA, Italian National Agency for New Technologies, Energy and Sustainable Economic Development, Casaccia Research Centre, Materials and Physicochemical Processes Technical Unit (SSPT-PROMAS-MATPRO), Via Anguillarese 301, 00123 Rome, Italy; mariangela.bellusci@enea.it (M.B.); elisabetta.simonetti@enea.it (E.S.); massimo.defrancesco@enea.it (M.D.F.)

* Correspondence: gianni.appetecchi@enea.it; Tel.: +39-06-3048-3924

Received: 31 July 2020; Accepted: 9 September 2020; Published: 11 September 2020

\section{Featured Application: Sodium battery systems.}

\begin{abstract}
Na}^{+}$-conducting, binary electrolytic mixtures, based on 1-ethyl-3-methyl-imidazolium, trimethyl-butyl-ammonium, and $\mathrm{N}$-alkyl- $\mathrm{N}$-methyl-piperidinium ionic liquid (IL) families, were designed and investigated. The anions were selected among the per(fluoroalkylsulfonyl)imide families. Sodium bis(trifluoromethylsulfonyl)imide, NaTFSI, was selected as the salt. The NaTFSI-IL electrolytes, addressed to safer sodium battery systems, were studied and compared in terms of ionic conductivity and thermal stability as a function of the temperature, the nature of the anion and the cation aliphatic side chain length. Room temperature conductivities of interest for sodium batteries, i.e., largely overcoming $10^{-4}$ or $10^{-3} \mathrm{~S} \mathrm{~cm}^{-1}$, are displayed. Similar conduction values are exhibited by the EMI-based samples even below $-10^{\circ} \mathrm{C}$, making these electrolyte mixtures potentially appealing also for low temperature applications. The NaTFSI-IL electrolytes, with the exception of the FSI-ones, are found to be thermally stable up to $275^{\circ} \mathrm{C}$, depending on the nature of the cation and/or anion, thus extending their applicability above $100^{\circ} \mathrm{C}$ and remarkably increasing the reliability and safety of the final device, especially in the case of prolonged overheating.
\end{abstract}

Keywords: sodium electrolytes; ionic liquids; sodium batteries

\section{Introduction}

The sodium-ion battery technology is a promising alternative to the currently market-dominating lithium-ion one due to the much larger availability, the lower cost and the similar redox potential ( $2.71 \mathrm{~V}$ vs. $\mathrm{H}+/ \mathrm{H} 2)$ of $\mathrm{Na}$ with respect to $\mathrm{Li}[1,2]$. Analogously to the lithium ones, the sodium-ion devices use electrolytes based on hazardous and volatile organic solvents, thus representing severe safety issues especially in case of upscaled systems and electric/thermal abuse [3]. In this scenario, ionic liquids (ILs), salts molten at room temperature or below, are appealing substitutes as safer (due to their exceptional flame retarding properties, negligible vapor pressure, thermal robustness) electrolytic solvents for replacing the organic ones [4]. Ionic liquids, constituted by pyrrolidinium cations and per(fluoroalkylsulfonyl)imide anions, have been successfully investigated as electrolyte components for $\mathrm{Na}^{+}$battery systems [5-9]. However, the physicochemical investigations were restricted to a very limited number of IL cations (mainly pyrrolidinium) and anions (mainly, bis(trifluoromethylsulfonyl)imide) and, therefore, only few ionic liquid types were studied until now for being addressed to sodium devices.

In the present paper, we show the results coming from investigations carried out on $\mathrm{Na}^{+}$-conducting electrolytes based on different IL families which, to our knowledge, have been 
never reported in the literature for applications in the sodium battery technology. For instance, ionic liquids, obtained combing 1-ethyl-3-methyl-imidazolium (EMI), trimethyl-butyl-ammonium $\left(\mathrm{N}_{1114}\right)$, and $N$-alkyl-N-methyl-piperidinium ( $\left.\mathrm{PIP}_{1 \mathrm{~A}}\right)$ cations with bis(fluorosulfonyl)imide (FSI), bis(trifluoromethylsulfonyl)imide (TFSI) and (nonafluorobutylsulfonyl)(trifluoromethylsulfonyl) imide $\left(\mathrm{IM}_{14}\right)$ anions, were synthesized and studied in the presence of the NaTFSI salt. The NaTFSI-IL sodium electrolytes were qualified in terms of ion transport properties and thermal stability.

\section{Materials and Methods}

The ionic liquids were synthesized and purified through an eco-friendly route detailly described elsewhere [10,11]. The reagents, namely $N$-methyl-imidazole (Sigma-Aldrich, Saint Louis, MO, USA, 99\%), N-methyl-piperidine (Sigma-Aldrich, 99\%), bromoethane (Sigma-Aldrich, 98\%), 1-bromopropane (Sigma-Aldrich, 99\%), 1-bromobutane (Sigma-Aldrich, 99\%), sodium bis(fluorosulfonyl)imide (NaFSI, Solvionic, Toulouse, France, $>99.9 \%$ ), lithium bis(trifluoromethylsulfonyl)imide (LiTFSI, 3M, Saint Paul, MN, USA, >99.9\%) and (nonafluorobutylsulfonyl)(trifluoromethylsulfonyl)imide acid ( $\mathrm{HIM}_{14}, 3 \mathrm{M}$, $60 \mathrm{wt} . \%$ in water solution) were used as received whereas the trimethylbutylammonium bromide $\left(\mathrm{N}_{1114} \mathrm{Br}\right.$, Solvionic, $\left.98 \%\right)$ precursor was purified through sorbents materials [11], i.e., activated carbon (Sigma-Aldrich, Darco-G60) and acidic alumina (Sigma-Aldrich, Brockmann I). The latter were previously treated as reported in ref. [11]. Deionized ultrapure water (Milli-Q, Millipore, Billerica, MA, USA) was used as the only processing solvent.

The 0.1NaTFSI-0.9IL (where 0.1 and 0.9 indicate the mole fractions) [9] electrolytes were prepared by quickly dissolving $\left(\right.$ at $50{ }^{\circ} \mathrm{C}$ ) the proper amount of the NaTFSI (Solvionic, $>99.9 \%$, previously vacuum dried overnight at $120^{\circ} \mathrm{C}$ ) salt in the ionic liquids.

The water content was determined (coulombometric titrations) by an automatic Karl Fischer titrator (Metrohm, Columbus, OH, USA) within a controlled atmosphere dry room (moisture level $<10 \mathrm{ppm}$ ). The content of halide and lithium was checked by X-ray fluorescence (Shimadzu energy dispersion EDX-720 spectrometer, Kyoto, Japan) and atomic absorption analysis (Varian SpetcrAA model 220 atomic absorption spectrometer, SpectraLab, Markham, ON, Canada), respectively.

The ionic conductivity of the NaTFSI-IL binary electrolytes was determined (through a conductivity meter Amel 160, AMEL, Milan, Italy) by loading (in the dry room) the samples in sealed, glass cells (Amel 192/K1, AMEL, cell constant equal to $1.00 \pm 0.01 \mathrm{~cm}$ ) equipped with two porous Pt electrodes. The measurements were carried out in the temperature range from -40 to $80^{\circ} \mathrm{C}$ (Binder GmbH MK53 climatic test chamber, BINDER, Tuttlingen, Germany). To fully crystallize the IL electrolytes [9], the cells were immersed in liquid nitrogen for about one minute and then immediately transferred in the climatic chamber at $-40^{\circ} \mathrm{C}$. This route was repeated until the ionic liquid samples kept solid (at $-40^{\circ} \mathrm{C}$.) After a storage period at $-40^{\circ} \mathrm{C}$ for at least $24 \mathrm{~h}$, the conductivity of the sodium IL electrolytes was measured by running a heating scan at $1^{\circ} \mathrm{Ch}^{-1}$.

The thermal stability of the NaTFSI-IL electrolytes was evaluated in argon atmosphere (100 $\mathrm{mL} \mathrm{min}^{-1}$ flow) through isothermal thermo-gravimetrical analysis (TGA) measurements at different temperature steps by using a Pyris Diamond analyzer (Perkin Elmer, Waltham, MA, USA). A very fast ramp $\left(40^{\circ} \mathrm{C} \mathrm{min}^{-1}\right)$ was set up for adjusting the temperature step by step, i.e., the time associated to the temperature ramp between two consecutive (temperature) steps, differing in $25^{\circ} \mathrm{C}$, is extremely short (just a few seconds). This procedure allowed to give more reliable data with respect to those obtained from ramp temperature measurements [9]. The samples (5-10 $\mathrm{mg}$ ) were placed in sealed, pin-holed, aluminum pans. The TGA measurements have been repeated, on each IL electrolyte sample, at least twice to verify the reproducibility of the results.

\section{Results and Discussion}

The investigated ionic liquid electrolytes have exhibited a purity level above $99.5 \mathrm{wt} . \%$; in particular, the content of lithium, halide and moisture is below $2 \mathrm{ppm}$. 
The ion transport properties have been studied as a function of the temperature and the obtained results are reported in Figure 1a (EMI-based electrolytes), $1 \mathrm{~b}\left(\mathrm{~N}_{11114}\right.$-based) and 1c ( $\mathrm{PIP}_{1 \mathrm{~A}}$-based), respectively. The conductivity-temperature diagram of the 0.1NaTFSI-0.9IL electrolytes containing TFSI-based ionic liquids (EMITFSI, $\mathrm{N}_{1114}$ TFSI, PIP 14 TFSI) exhibits ion conduction values around $10^{-7} \mathrm{~S} \mathrm{~cm}^{-1}$ (i.e., typical of a crystalline solid state) $[4,5,9]$ from $-40{ }^{\circ} \mathrm{C}$ up to the melting point, evidenced by a step increase (from two to three orders of magnitude) of the ionic conductivity. Conversely, the mixtures containing FSI-based ILs (EMIFSI, $\mathrm{N}_{1114} \mathrm{FSI}$ ) show, in the low-temperature range, an almost linear increase in conduction, likely attributable to an ion mobility gain due to solid-solid phase transitions occurring prior the melting [12]. A similar trend is also observed for the 0.1NaTFSI-0.9PIP 13 TFSI sample, in agreement with the conductivity results reported elsewhere for pure PIP 13 TFSI [13]. A two-orders-of-magnitude jump (not registered for the FSI-based electrolytes) is, however, detected around the melting temperature. A comparison among the conductivity-temperature plots evidences that solid-liquid transitions, recorded in the FSI-based electrolytes, occur at lower temperatures with respect to those observed in the TFSI ones. The melting point of an IL material is determined by the strength of its crystal lattice which, in turn, is controlled by competitive factors, such as surface charge density, intermolecular forces (Van der Waals) and molecular symmetry [14], which have opposite effect. Therefore, the lower Van der Waals forces, due to the smaller steric hindrance of the FSI anion, might counterbalance the higher surface charge density (which, conversely, tends to increase the melting point), thus decreasing the melting temperature with respect to the TFSI electrolytes [4].
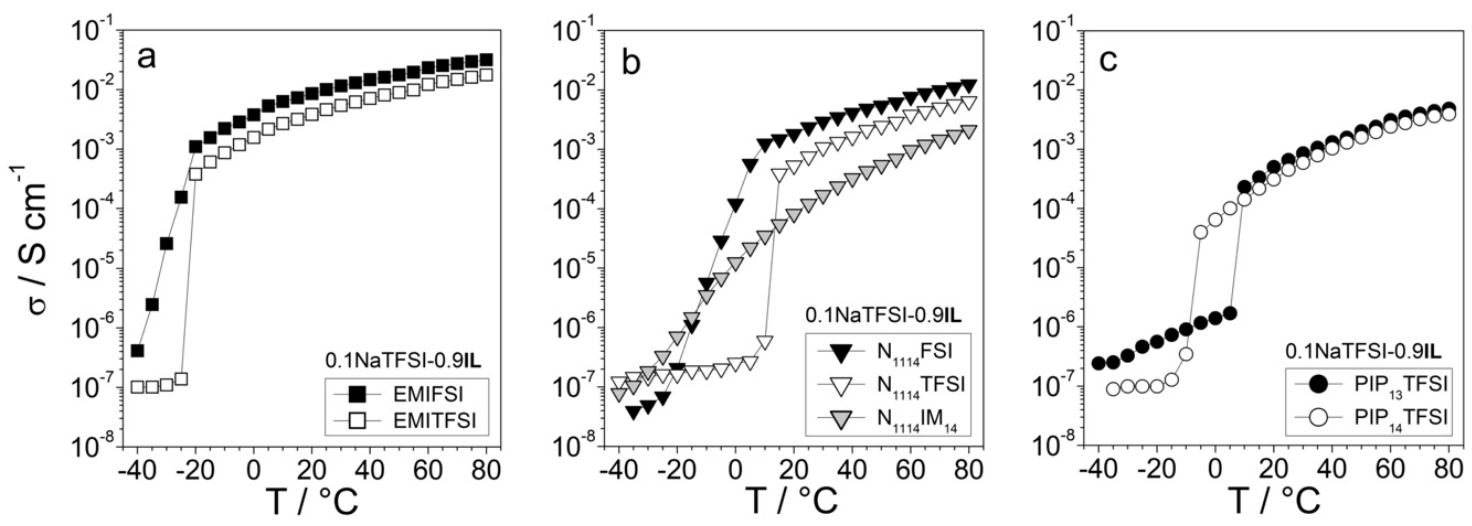

Figure 1. Ionic conductivity vs. temperature dependence of 0.1NaTFSI-0.9IL sodium electrolytes: (a) EMI-based electrolytes; (b) $\mathrm{N}_{1114}$-based electrolytes; (c) PIP $1 \mathrm{~A}$-based electrolytes.

No sudden conductivity raise is seen for the $0.1 \mathrm{NaTFSI}-0.9 \mathrm{~N}_{1114} \mathrm{IM}_{14}$ electrolyte, indicating the absence of any solid-liquid phase transition in the investigated temperature range. This is likely attributable to the high asymmetry of the $\mathrm{IM}_{14}$ anion with respect to FSI and TFSI (symmetrical), which strongly hinders the ion packing and, therefore, the IL crystallization. In the molten state, the 0.1NaTFSI-0.9IL samples display a VTF behavior, typical of the ionic liquid electrolytes [4]. Further increases in temperature beyond that of the melting lead to a progressive raise in conductivity, due to the convective thermal motions within the ionic liquid media. The ionic conduction values, in the liquid state, progressively decrease with increasing the steric footprint of the anion in the following order: FSI > TFSI > IM 14 . This behavior, reported in the literature for other IL electrolytes [4], is due to the progressive increase in viscosity (linked to the size of the ions), which slows down the mobility of the ions within the IL material [4].

In the molten state, a moderate decrease in conduction is observed for the $0.1 \mathrm{NaTFSI}-0.9 \mathrm{PIP}{ }_{14}$ TFSI electrolyte with respect to $0.1 \mathrm{NaTFSI}-0.9 \mathrm{PIP}_{13} \mathrm{TFSI}$, attributable to the longer cation alkyl chain (n-butyl compared to n-propyl), which increases the steric bulk and, therefore, the viscosity of the piperidinium IL [13]. Conversely, the increase in alkyl chain length leads to a greater asymmetry of the cation, 
hindering the formation of the crystalline lattice and, therefore, producing a decrease in the melting temperature of the ionic liquid, i.e., the PIP 14 TFSI electrolyte exhibits a melting point of approximately $20{ }^{\circ} \mathrm{C}$ lower than that of the $\mathrm{PIP}_{13} \mathrm{TFSI}$ one, once more in agreement with the results obtained for the pure ILs [13].

The ionic conductivity data, determined at selected temperatures, of the $0.1 \mathrm{NaTFSI}-0.9 \mathrm{IL}$ sodium electrolytes are summarized in Table 1 . At $-20^{\circ} \mathrm{C}$ only the EMI-based samples exhibit conduction values (i.e., from $10^{-4}$ to $10^{-3} \mathrm{~S} \mathrm{~cm}^{-1}$ ) of interest for practical devices, making these electrolytes appealing for low temperature applications. At $20^{\circ} \mathrm{C}$ all investigated NaTFSI-IL electrolytes, with the exception of the NaTFSI- $0 \mathrm{~N}_{1114} \mathrm{IM}_{14}$ sample, largely overcome $10^{-4}$ or $10^{-3} \mathrm{~S} \mathrm{~cm}^{-1}$. The EMI-based samples display higher conductivity values than those containing $\left(\mathrm{N}_{1114}\right)^{+}$and piperidinium cations, due to the lower viscous drag ascribable to the smaller size and planar configuration of the imidazolium cation [4]. At $80^{\circ} \mathrm{C}$, the conductivity value of all sodium IL electrolytes largely exceeds $10^{-3} \mathrm{~S} \mathrm{~cm}^{-1}$; in particular, that of EMI- and FSI-based electrolytes widely overcomes $10^{-2} \mathrm{~S} \mathrm{~cm}^{-1}$.

Table 1. Thermal stability and ionic conductivity $(\sigma)$ values of 0.1 NaTFSI-0.9IL sodium electrolytes.

\begin{tabular}{cccccc}
\hline \multirow{2}{*}{$\begin{array}{c}\text { Ionic } \\
\text { Liquid }\end{array}$} & \multirow{2}{*}{$\begin{array}{c}\text { Thermal } \\
\text { Stability/ }{ }^{\circ} \mathbf{C}\end{array}$} & \multicolumn{4}{c}{$\boldsymbol{\sigma} / \mathbf{S ~ c m}^{-\mathbf{1}}$} \\
\cline { 3 - 6 } & & $\mathbf{- 2 0} \mathbf{C}$ & $\mathbf{2 0} \mathbf{C}$ & $\mathbf{5 0}{ }^{\circ} \mathbf{C}$ & $\mathbf{8 0}^{\circ} \mathbf{C}$ \\
\hline EMIFSI & 150 & $(1.1 \pm 0.1) \times 10^{-3}$ & $(8.6 \pm 0.5) \times 10^{-3}$ & $(1.8 \pm 0.1) \times 10^{-2}$ & $(3.2 \pm 0.2) \times 10^{-2}$ \\
EMITFSI & 275 & $(3.8 \pm 0.2) \times 10^{-4}$ & $(3.8 \pm 0.2) \times 10^{-3}$ & $(8.9 \pm 0.5) \times 10^{-3}$ & $(1.8 \pm 0.2) \times 10^{-2}$ \\
$\mathrm{~N}_{1114}$ FSI & 150 & $(2.8 \pm 0.2) \times 10^{-7}$ & $(1.8 \pm 0.1) \times 10^{-3}$ & $(5.4 \pm 0.3) \times 10^{-3}$ & $(1.2 \pm 0.1) \times 10^{-2}$ \\
$\mathrm{~N}_{1114}$ TFSI & 225 & $(1.6 \pm 0.1) \times 10^{-7}$ & $(5.3 \pm 0.3) \times 10^{-4}$ & $(2.5 \pm 0.2) \times 10^{-3}$ & $(6.5 \pm 0.4) \times 10^{-3}$ \\
$\mathrm{~N}_{1114}$ IM & 225 & $(7.0 \pm 0.4) \times 10^{-7}$ & $(8.2 \pm 0.5) \times 10^{-5}$ & $(5.5 \pm 0.3) \times 10^{-4}$ & $(2.1 \pm 0.1) \times 10^{-3}$ \\
$\mathrm{PIP}_{13}$ TFSI & 250 & $(5.6 \pm 0.3) \times 10^{-7}$ & $(5.0 \pm 0.3) \times 10^{-4}$ & $(2.0 \pm 0.1) \times 10^{-3}$ & $(4.9 \pm 0.3) \times 10^{-3}$ \\
$\mathrm{PIP}_{14}$ TFSI & 225 & $(9.9 \pm 0.5) \times 10^{-8}$ & $(3.1 \pm 0.2) \times 10^{-4}$ & $(1.6 \pm 0.1) \times 10^{-3}$ & $(3.9 \pm 0.2) \times 10^{-3}$ \\
\hline
\end{tabular}

The results, obtained from isothermal TGA measurements, are illustrated in Figure 2a (samples containing the FSI anion) and Figure $2 \mathrm{~b}$ (TFSI and $\mathrm{IM}_{14}$ anions), respectively. The time evolution of the sample temperature is highlighted by the stepped profiles (grey traces). All investigated samples show an initial loss in weight ranging from $0.4 \%$ to $0.6 \%$ due to the removal of absorbed $\mathrm{H}_{2} \mathrm{O}$ (accentuated by the presence of the NaTFSI salt) during handling (sample loading into the pans).
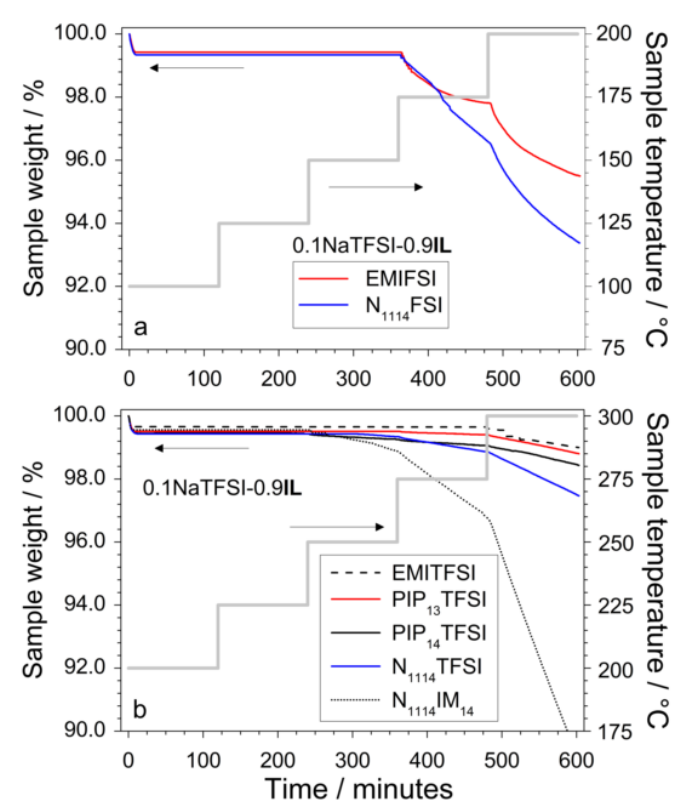

Figure 2. Isothermal TGA trace of 0.1NaTFSI-0.9IL sodium electrolytes: (a) FSI-based electrolytes; (b) TFSI- and $\mathrm{IM}_{14}$-based electrolytes. The sample temperature vs. time profile is represented by the stepwise grey trace. 
The binary mixtures containing the FSI anion exhibit thermal stability up to $150{ }^{\circ} \mathrm{C}$ and reveal a progressive weight loss above this temperature (more accentuated in the passage from 175 to $200^{\circ} \mathrm{C}$ ). The EMIFSI electrolyte shows a lower degradation than that containing $\mathrm{N}_{1114} \mathrm{FSI}$, due to the higher stability of the (EMI) ${ }^{+}$cation compared to $\left(\mathrm{N}_{1114}\right)^{+}$[4]. This relatively low thermal robustness, with respect to other IL families, is ascribable to the higher reactivity of the -SO2F group (in particular, the S-F bond) toward water and nucleophiles, especially at elevated temperatures [15-17]. Among the electrolyte mixtures containing the TFSI and $\mathrm{IM}_{14}$ anions (Figure $2 \mathrm{~b}$ ), the EMITFSI sample displays the highest thermal stability: no weight loss is observed up to $275^{\circ} \mathrm{C}$ whereas, after $2 \mathrm{~h}$ of heating at $300^{\circ} \mathrm{C}$, the residual mass of sample still exceeds $99 \%$. The NaTFSI-N ${ }_{1114}$ TFSI and NaTFSI-N ${ }_{1114} \mathrm{IM}_{14}$ mixtures exhibit thermal stability up to $225^{\circ} \mathrm{C}$ : above this temperature, however, a more pronounced degradation is seen for the $\mathrm{N}_{1114} \mathrm{IM}_{14}$-based electrolyte. This behavior might be attributed to a less difficult removal of the $-\mathrm{CF}_{2} \mathrm{CF}_{2} \mathrm{CF}_{2} \mathrm{CF}_{3}$ nonafluorobutyl chain (present in the anion $\mathrm{IM}_{14}$ anion) compared to the $-\mathrm{CF}_{3}$ trifluoromethyl one, even if this issue should be supported by further investigation. The piperidinium electrolyte samples (Figure $2 \mathrm{~b}$ ) are thermally stable up to 250 (PIP 13 TFSI) and $225^{\circ} \mathrm{C}$ (PIP 14 TFSI), respectively. A progressive weight loss is observed above these temperatures, more marked for the mixture containing PIP 14 TFSI. Therefore, the n-propyl chain seems to confer, compared to the n-butyl one, a greater resistance to the thermal degradation. These results are in good agreement with previous data [13], which show progressively decrease of the thermal stability with increasing the length of the main alkyl side chain of the piperidinium cation. To conclude, the results of the TGA measurements, summarized in Table 1, have highlighted a different thermal stability of the investigated NaTFSI-IL electrolytes, according to the following sequences: EMI $>\mathrm{PIP}_{13}>\mathrm{PIP}_{14}>\mathrm{N}_{1114}$ (cations); TFSI $>\mathrm{IM}_{14}$ $>$ FSI (anions).

\section{Conclusions}

To summarize, the 0.1NaTFSI-0.9IL mixtures, investigated in the present work, show potentially appealing characteristics as innovative ionic liquid electrolytes for realizing safer and more reliable sodium battery systems. Room temperature conductivities of interest for practical devices, i.e., largely overcoming $10^{-4}$ or $10^{-3} \mathrm{~S} \mathrm{~cm}^{-1}$, are displayed by all investigated IL mixtures. The EMI-based electrolytes are seen to exhibit the best ion transport properties, especially at low temperatures: at $-20^{\circ} \mathrm{C}$ from $10^{-4}$ to $10^{-3} \mathrm{~S} \mathrm{~cm}^{-1}$ are observed, this extending the applicability of these electrolytes even at low temperatures. The FSI mixtures display faster transport properties than the TFSI- and $\mathrm{IM}_{14}$-based ones. A conductivity well above $10^{-2} \mathrm{~S} \mathrm{~cm}^{-1}$ is approached at $80^{\circ} \mathrm{C}$.

The 0.1NaTFSI-0.9IL electrolytes were found to be thermally robust up to $275^{\circ} \mathrm{C}$ (EMITFSI-based electrolyte) with the exception of the FSI-based ones (up to $150^{\circ} \mathrm{C}$ ). This important peculiarity enables these sodium electrolytes to be used in battery systems operating at temperatures (i.e., $>100{ }^{\circ} \mathrm{C}$ ) which are not allowed in standard organic solutions. At the same time, in conjunction with the flame-retardant properties and the very low volatility of the ionic liquid components, it strongly increases the reliability and the safety of the device, particularly in the case of prolonged overheating.

Author Contributions: Investigation, validation, M.B.; investigation, validation, E.S.; investigation, M.D.F.; conceptualization, methodology, data curation, supervision, writing, review, editing, G.B.A. All authors have read and agreed to the published version of the manuscript.

Funding: This research was funded by the Italian Ministry of the Economic Development (MISE) within the ENEA-MISE Program Agreement on Electric System Research.

Conflicts of Interest: The authors declare no conflict of interest.

\section{References}

1. Wang, A.; Hu, X.; Tang, H.; Zhang, C.; Liu, S.; Yang, Y.; Yang, Q.; Luo, J. Processable and moldable sodium metal anodes. Angew. Chem. Int. Ed. 2017, 56, 11921-11926. [CrossRef] [PubMed]

2. Kumar Nayak, P.; Yang, L.; Brehm, W.; Adelhelm, P. From lithium-ion to sodium-ion batteries: A materials perspective. Angew. Chem. Int. Ed. 2018, 57, 102-120. [CrossRef] [PubMed] 
3. Bandhauer, T.M.; Garimella, S.; Fuller, T.F. A critical review of thermal issues in lithium-ion batteries. J. Electrochem. Soc. 2011, 158, R1-R25. [CrossRef]

4. Appetecchi, G.B.; Montanino, M.; Passerini, S. Ionic Liquid-Based Electrolytes for High-Energy Lithium Batteries. In Ionic Liquids: Science and Applications; Visser, A.E., Bridges, N.J., Rogers, R.D., Eds.; American Chemical Society: Washington, DC, USA, 2013; pp. 67-128.

5. Serra Moreno, J.; Maresca, G.; Panero, S.; Scrosati, B.; Appetecchi, G.B. Sodium-conducting ionic liquid-based electrolytes. Electrochem. Commun. 2014, 43,1-4. [CrossRef]

6. Wongittharom, N.; Lee, T.-C.; Wang, C.-H.; Wang, Y.-C.; Chang, J.-K. Electrochemical performance of $\mathrm{Na} / \mathrm{NaFePO} 4$ sodium-ion batteries with ionic liquid electrolytes. J. Mater. Chem. A 2014, 2, 5655-5661. [CrossRef]

7. Hasa, I.; Passerini, S.; Hassoun, J. Characteristics of anionic liquid electrolyte for sodium-ion batteries. J. Power Sources 2016, 303, 203-207. [CrossRef]

8. Forsyth, M.; Yoon, H.; Chen, F.; Zhu, H.; MacFarlane, D.R.; Armand, M.; Howlett, P.C. Novel Na ${ }^{+}$ion diffusion mechanism in mixed organic-inorganic ionic liquid electrolyte leading to high $\mathrm{Na}^{+}$transference number and stable, high rate electrochemical cycling of sodium cells. J. Phys. Chem. C 2016, 120, 4276-4286. [CrossRef]

9. Brutti, S.; Navarra, M.A.; Maresca, G.; Panero, S.; Manzi, J.; Simonetti, E.; Appetecchi, G.B. Ionic liquid electrolytes for room temperature sodium battery systems. Electrochim. Acta 2019, 306, 317-326. [CrossRef]

10. Montanino, M.; Alessandrini, F.; Passerini, S.; Appetecchi, G.B. Water-based synthesis of hydrophobic ionic liquids for high-energy electrochemical devices. Electrochim. Acta 2013, 96, 124-133. [CrossRef]

11. De Francesco, M.; Simonetti, E.; Giorgi, G.; Appetecchi, G.B. About purification route of hydrophobic ionic liquids. Challenges 2017, 8, 11. [CrossRef]

12. Paillard, E.; Zhou, Q.; Henderson, W.A.; Appetecchi, G.B.; Montanino, M.; Passerini, S. Electrochemical and physicochemical properties of PY ${ }_{14}$ FSI-based electrolytes with LiFSI. J. Electroch. Soc. 2009, 156, A891-A895. [CrossRef]

13. Montanino, M.; Carewska, M.; Alessandrini, F.; Passerini, S.; Appetecchi, G.B. The role of the aliphatic side chain on $\mathrm{N}$-alkyl-N-alkylpiperidinium bis(trifluoromethansulfonyl)imide ionic liquids. Electrochim. Acta 2011, 57, 153-159. [CrossRef]

14. Dearden, J.C. The QSAR prediction of melting point, a property of environmental relevance. Sci. Total Environ. 1991, 109, 59-68. [CrossRef]

15. Hallac, B.B.; Geiculescu, O.E.; Rajagopal, R.V.; Creager, S.E.; DesMarteau, D.D. Advanced fluoride-based materials for energy conversion. Electrochim. Acta 2008, 53, 5985-5991. [CrossRef]

16. Toulgoat, J.F.; Langlois, B.R.; Medebielle, M.; Sanchez, J.-Y. An efficient preparation of new sulfonyl fluorides and lithium sulfonates. J. Org. Chem. 2007, 72, 9046-9052. [CrossRef] [PubMed]

17. Toulgoat, J.F.; Langlois, B.R.; Medebielle, M.; Sanchez, J.-Y. Efficient preparation of new fluorinated lithium and ammonium sulfonimides. J. Org. Chem. 2008, 73, 5613. [CrossRef] [PubMed]

(C) 2020 by the authors. Licensee MDPI, Basel, Switzerland. This article is an open access article distributed under the terms and conditions of the Creative Commons Attribution (CC BY) license (http://creativecommons.org/licenses/by/4.0/). 